\title{
Evaluation of an Australian indigenous housing programme: community level impact on crowding, infrastructure function and hygiene
}

\author{
Ross S Bailie, ${ }^{1}$ Elizabeth L McDonald, ${ }^{1}$ Matthew Stevens, ${ }^{1}$ Steven Guthridge, ${ }^{2}$ \\ David R Brewster ${ }^{3}$
}

- Supplementary tables are published online only. To view these files please visit the journal online (http://jech.bmj. com)

${ }^{1}$ Menzies School of Health Research, Institute of Advanced Studies, Charles Darwin University, Darwin, Northern Territory, Australia ${ }^{2}$ Health Gains Planning Division, Northern Territory Government Department of Health and Families, Darwin, Northern Territory, Australia

${ }^{3}$ James Cook University, School of Medicine, Cairns, Queensland, Australia

Correspondence to Ross S Bailie, Post Office Box 41096, Casuarina, North Territory 0811, Australia; ross.bailie@menzies.edu.au

Accepted 8 January 2010 Published Online First 12 May 2010

\section{ABSTRACT}

Background and Aim Housing programmes in indigenous Australian communities have focused largely on achieving good standards of infrastructure function. The impact of this approach was assessed on three potentially important housing-related influences on child health at the community level: (1) crowding, (2) the functional state of the house infrastructure and (3) the hygienic condition of the houses

Methods A before-and-after study, including house infrastructure surveys and structured interviews with the main householder, was conducted in all homes of young children in 10 remote Australian indigenous communities. Results Compared with baseline, follow-up surveys showed (1) a small non-significant decrease in the mean number of people per bedroom in the house on the night before the survey $(3.4,95 \% \mathrm{Cl} 3.1$ to 3.6 at baseline vs $3.2,95 \% \mathrm{Cl} 2.9$ to 3.4 at follow-up; natural logarithm transformed test, $t=1.3, p=0.102$ ); (2) a marginally significant overall improvement in infrastructure function scores (Kruskal-Wallis test, $\chi^{2}=3.9, p=0.047$ ); and (3) no clear overall improvement in hygiene (Kruskal-Wallis test, $\chi^{2}=0.3, p=0.605$ ).

Conclusion Housing programmes of this scale that focus on the provision of infrastructure alone appear unlikely to lead to more hygienic general living environments, at least in this study context. A broader ecological approach to housing programmes delivered in these communities is needed if potential health benefits are to be maximised. This ecological approach would require a balanced programme of improving access to health hardware, hygiene promotion and creating a broader enabling environment in communities.

\section{INTRODUCTION}

Despite health improvement being an explicit purpose of housing programmes in indigenous communities, ${ }^{1-3}$ the housing conditions and health of many indigenous Australians continue to be very poor. ${ }^{4}$ Australian government policy initiatives have consistently attempted to improve hygiene and living conditions through infrastructure programmes. ${ }^{5}$ Attention has been focused on the components of infrastructure required for the effective conduct of 'healthy living practices' (HLPs). ${ }^{6}$ There is mounting evidence that the focus on infrastructure and relative neglect of hygiene and other interventions has failed to produce improvements in health in Australia ${ }^{7-11}$ and internationally. ${ }^{12-14}$

Internationally, the contribution of poor domestic hygiene to poor health is well recognised, ${ }^{15} 16$ as is household crowding. ${ }^{16-24}$ These factors may have a significant influence at the general community level as well as at the household level. However, the tendency for epidemiological research to focus on individual child or household level exposures and outcomes of housing improvements means there is little understanding of the extent to which reduced risks to health may be moderated by the extent to which housing programmes impact at the general community level. This moderating effect is likely to be an important influence on the potential for health improvement where there is a high level of interaction between children from different households and mobility of children between households.

This paper addresses this issue of community level impact of housing programmes by reporting on three potentially important housing-related factors affecting child health: (1) overcrowding; (2) the functional state of the house infrastructure; and (3) the hygienic condition of houses. This work is part of a wider study of housing and child health called the Housing Improvement and Child Health ( $\mathrm{HICH})$ Study.

\section{Study setting and intervention}

The HICH Study was conducted in 10 Northern Territory (NT) communities in which there was the greatest construction of new houses by the Australian government's National Aboriginal Health Strategy (NAHS) Environmental Health Program and other large infrastructure programmes over the period 2004-5. The communities were spread across the NT, and their distance from the nearest regional town ranged between 1 and $500 \mathrm{~km}$. Four of the 10 communities were isolated by floodwaters for at least part of most years. Indigenous people living in these communities generally experience poor health, educational, employment and other social outcomes. ${ }^{25}$ The mean population of the 10 communities was 730 (range 250-1450) and the average number of houses in each community was 66 (range 33-103). The average of 11 people per house in these communities is markedly higher than the national average for indigenous households of 3.5 and the average for all Australian households of 2.6. ${ }^{26}$ The high levels of crowding and multi-family households means that, when new houses become available in these remote communities, there is a shift from pre-existing to new houses and between pre-existing houses. The extended nature of indigenous families means that this shift cannot be simply described in terms of 
rehousing of clearly-defined family units. Allocation of new houses is reportedly based on need and residential history, ${ }^{26}$ but the definition of need and the way the allocation criteria are applied is unclear. Some members of a pre-existing household may move to a new house, and/or some may move to another pre-existing house, and/or some may stay in the same house. These moves may or may not be associated with a change in a child's primary carer.

The intervention in this study was the construction of additional housing to specific housing standards for these communities. These standards are significantly more rigorous than standards applied in these communities over past decades. ${ }^{27}$ The average number of new houses to be constructed in each of the 10 communities was 11 (range 7-15). A small number of uninhabitable houses were earmarked for demolition. There were no concurrent renovation programmes or hygiene promotion activities conducted over the study period, so the housing intervention essentially consisted of the construction of a defined number of new houses.

\section{METHODS}

The conceptual framework and methods used in the $\mathrm{HICH}$ Study have been described elsewhere. ${ }^{28}$ The main methods relevant to this paper include: (1) structured interviews with the main householder in all houses with children aged $\leq 7$ years; (2) infrastructure surveys of these houses; and (3) interviews with staff of the community housing office and/or council and inspection of their housing records. Local community residents were employed to work with survey teams and played a key role in the identification of houses where there were children aged $<7$ years.

The baseline householder interviews and infrastructure surveys were completed in each study community on average 6 months (range 1-18) before occupation of new houses. Follow-up interviews and surveys were completed on average 10 months (range 7-12) after occupation of new houses. The total population in each study community did not change significantly between baseline and follow-up.

Our assessment of the impact of the building programme on the housing conditions for young children is based on comparison of conditions in houses that were the usual place of residence for children for whom we were able to collect data at both baseline and follow-up.

The infrastructure surveys involved an inspection (and, where appropriate, testing) of the functional state of each house in relation to the effective conduct of HLPs, including required infrastructure items based on the NT minimum housing standards ${ }^{29}$ and national indigenous housing guidelines. ${ }^{30}$ In order to provide more specific measures of infrastructure related to risks to child health, the nine HLPs specified in the national guidelines were refined for the purpose of this study. The HLP of 'reducing negative contact between people and animals, insects and vermin' was split into two measures: 'separate dogs and people' and 'control pests and vermin'. Additional measures introduced were: 'control mould', as an observable effect of heat and humidity related to the HLP of 'control temperature'; and 'sleeping and bedding', as a measure of the key requirement for a space for children to get adequate rest.

Two methods were used to score house infrastructure function. The first method, the Failed Healthy Living Practices (FHLPs) score, was based on an assessment of all items of infrastructure required for conducting each HLP as described in our previous work ${ }^{20}$ and in table 1 in the online supplement. For any HLP for which an item of infrastructure was not func- tioning at an adequate level (fully functional or requiring minor maintenance only), the infrastructure required for that HLP was scored as failed. The overall assessment of house function using this method is then based on the number of failed HLPs. This measure was suited to assessment of infrastructure for eight of the HLPs. The overall FHLP score reflects the number of HLPs for which the score was 'fail' (potential range 0-8).

The 'technical function' approach of the FHLP score allows for an objective assessment of function for some HLPs. This has limitations in terms of practical function for the purposes of conducting healthy living practices and is not suited to assessment of all HLPs. We therefore used a complementary method to assess infrastructure function, the Surveyor Function Score (SFS). This relied on the surveyor using a 7-point Likert scale to provide a score of their perception of the functional state of the house in relation to the ability to conduct each HLP. The intention of this method was to address some of the limitations associated with the approach used for the FHLP score, to provide a finer measure of functional state (using a 1-7 score rather than a pass/fail assessment) and to include assessment of availability of other requirements (such as furnishings and fixtures) for the effective conduct of HLPs. Criteria for the SFS are shown in table 2 in the online supplement. A limitation of the SFS is that it is possibly a more subjective measure, hence its use in addition to rather than in place of the FHLP score.

To assess the hygienic condition of houses, surveyors were required to provide a Surveyor Condition Score (SCS) for the hygienic condition of the areas or infrastructure items required for specific HLPs and for the overall house using a 7-point Likert scale (see table 2 in the online supplement).

The repeatability of the SFS and SCS was examined through repeat surveys of 15 houses at least 1 week after the initial survey. ${ }^{25}$ Household hygiene was expected to vary over time in relation to cleaning and other household activities. Repeatability of the SCS provides an indication of the extent to which the SCS reflects the general state of household hygiene over time. On testing repeatability of the exact scores on the 1-7 scale, 9 of the 13 SCS measures recorded fair to moderate agreement $(0.20 \leq \kappa<0.60)$ while 5 (wash people, wash clothes and bedding, house surrounds, control pests and vermin, and overall house condition) showed slight agreement $(0.01 \leq \kappa<0.20)$. Across all of the SCS measures in the repeat survey, the scores were within one point of the original score in over $85 \%$ of cases. Repeatability across SFS measures would be expected to be relatively stable over a short time frame. Eleven of the 13 measures recorded fair to substantial agreement, with food preparation and storage, remove human waste and remove rubbish only showing a slight agreement. Across all of the SFS measures in the repeat survey, scores were within one point of the original score in over $87 \%$ of cases (see table 3 in online supplement).

Crowding, SFS, SCS and FHLP scores were compared between baseline and follow-up. The crowding data were not normally distributed and a natural logarithm transformation was applied prior to testing for a change over time using a t test. The Kruskal-Wallis test of equality of proportions was used to test for a change in overall SFS, SCS and FHLP scores. For individual SFSs and SCSs the non-parametric Kruskal-Wallis test was used to compare between baseline and follow-up, while a Fisher exact test was used for individual FHLPs.

\section{RESULTS}

At the time of the baseline surveys, 326 houses (51\%) were identified from a total of 644 houses in the 10 communities as being the main place of residence of at least one child aged 
$\leq 7$ years. The main householder in 285 (88\%) of these houses was interviewed. In 10 (3\%) houses the main householder declined to be interviewed and in 31 (11\%) houses the main householder was away on each of three consecutive visits by the project team. A total of 618 children living in these 285 houses were recruited to the study.

At the follow-up surveys we were able to identify 208 houses where children recruited at baseline were now living. These houses were the main place of residence for 418 (68\%) of the children originally recruited to the study. These 418 children make up the study cohort. They had been living in 185 houses at the time of the baseline surveys (figure 1).

Of the 208 houses where these children were living at followup, $44(21 \%)$ were new houses completed between the baseline and follow-up surveys.

Crowding data were available at baseline and follow-up for 327 (78\%) children. At follow-up there was a small non-significant decrease in the mean number of people per bedroom sleeping in the house on the night before the survey $(3.4,95 \% \mathrm{CI}$ 3.1 to 3.6 at baseline to $3.2,95 \%$ CI 2.9 to 3.4 at follow-up; natural logarithm transformed $t$ test, $t=1.3, p=0.102$ ). At baseline and follow-up surveys there was no significant change in the composition of households in terms of the numbers of younger and older children and adults.

\section{House infrastructure}

FHLP data were available at both baseline and follow-up for 315 children (75\%). The baseline distribution of the FHLP scores is skewed, peaking at scores of 6 for follow-up and 7 for baseline (a score of 7 means 7 out of the 8 infrastructure components scored using this method were scored as 'failed'). There is also a secondary peak at the score of 2 for follow-up (figure 2). The distribution shows a shift to improvement in scores, with no houses scoring 0 (a score of 0 means no failed infrastructure components) at baseline, and just under $5 \%$ scoring 0 at followup. The Kruskal-Wallis test showed a significant difference between baseline (mean 5.6, 95\% CI 5.3 to 6.0) and follow-up (4.4, $95 \%$ CI 4.1 to 4.8$)$ for the FHLP score $\left(\chi^{2}=22.8, p<0.001\right)$.

SFS data were available at both baseline and follow-up for 349 children (83\%) in the study cohort. The distribution of the SFS scores shows a bimodal pattern with peaks at scores of 2 and 4 for both time periods, suggesting some digit preference by the surveyors (figure 3 ). The distribution shows a shift to improvement in scores, with about $10 \%$ of houses receiving a score of 1 (best function) at follow-up compared with none at baseline. The Kruskal-Wallis test showed a marginally statistically significant difference between baseline (mean 3.8, 95\% CI 3.5 to 4.0) and follow-up (3.4, 95\% CI 3.1 to 3.6) for the SFS $\left(\chi^{2}=3.9\right.$, $\mathrm{p}=0.047)$. This reflects the same pattern of improvement seen in the FHLP data described above.

Changes between baseline and follow-up in the proportion of houses that failed specific HLPs or in the mean SFS score for specific HLPs are shown in tables 1 and 2. HLPs for which there was consistent evidence of improvement on both the FHLP score and SFS included: 'wash people', 'functioning toilet'/'remove human waste (toilet)' and 'wash clothes and bedding'. HLPs which were assessed by either the FHLP score or the SFS only and which showed evidence of improvement included: 'safe electrical', 'separate animals and humans', 'control pests and vermin', 'control mould', 'control temperature' and 'sleeping and bedding'. HLPs which were assessed on both the FHLP score and the SFS, but for which there was inconsistent evidence of improvement, included: 'prepare and store food' and 'control dust'. 'Reduce trauma', 'house surrounds' and 'overall house SFS
Figure 1 Crowding, house infrastructure and hygiene data at baseline and follow-up.

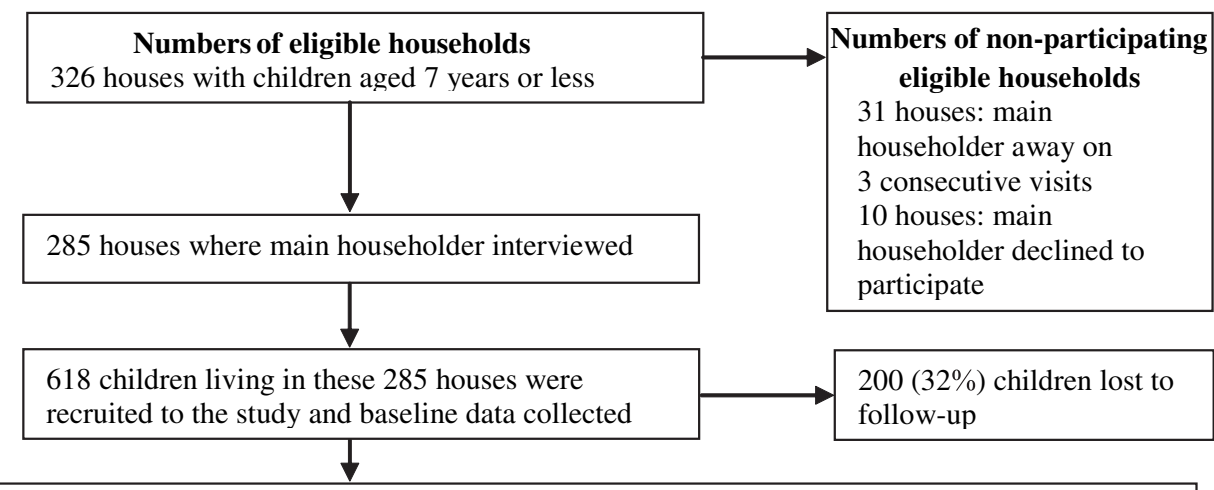

\section{Study cohort}

Comprises 418 (68\%) children followed up: living in 185 houses at baseline; 208 at follow-up. The householder in 153 (74\%) houses was the same person at baseline and follow-up.

\section{Crowding}

Data collected for 327 (78\%) children at baseline and follow-up, living in 146 (79\%) houses at baseline and $162(78 \%)$ houses at follow-up.

House infrastructure

FHLP $^{\mathrm{a}}$ and SFS ${ }^{\mathrm{b}}$ data

$315(75 \%)$ children living in: 138 (73\%) houses at baseline; and 153 (74\%) houses at follow-up.

House infrastructure/Hygiene

$\mathrm{SFS}^{\mathrm{b}}$ and $\mathrm{SCS}^{\mathrm{c}}$ data

$349(83 \%)$ children living in: $154(83 \%)$ houses at baseline; and $172(83 \%)$ houses at follow-up. 


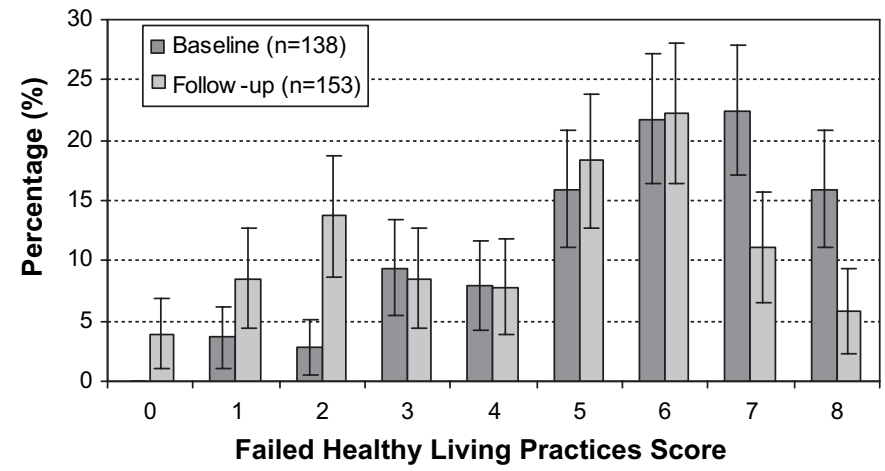

Figure 2 Distribution of Failed Healthy Living Practices score (with standard errors) for houses at baseline and follow-up.

were assessed on the SFS only and did not show evidence of improvement. 'Remove waste water' was assessed on the FHLP only and did not show evidence of improvement.

\section{Hygiene}

There is no evidence of improvement of the overall SCS at follow-up compared with baseline (figure 4). This is confirmed by the Kruskal-Wallis test (baseline mean 4.1, 95\% CI 3.9 to 4.4; follow-up mean 4.1, 95\% CI 3.9 to $4.4 ; \chi^{2}=0.3, p=0.605$ ). Kruskal-Wallis tests indicated a significant improvement in SCS scores for 'control mould' $\left(\chi^{2}=10.3, p=0.001\right)$ and 'control pests and vermin' ( $\left.\chi^{2}=4.9, p=0.028\right)$, and deterioration in 'prepare and store food' $\left(\chi^{2}=6.3, p=0.012\right.$; table 2$)$.

\section{DISCUSSION}

These empirical findings add to the Australian and international literature $^{7-11} 1314$ which indicates that programmes which are largely limited to improving the functional state of infrastructure have limited impact at the community level on major housing-related risks to health such as domestic hygiene. There was no systematic programme to achieve hygiene behaviour change in association with the building programme that was the subject of our study. Other possible contributing reasons for the lack of observable improvement in hygiene include: (1) the pattern of improvements in infrastructure function shows the improvements resulted largely from changes in the proportions of houses rated worst and best (figures 2 and 3). Thus, although there was a marked improvement in the functional state of houses for some children, at a population/community level there appears to have been little change; (2) the continuing high levels of crowding may have been a barrier to hygiene

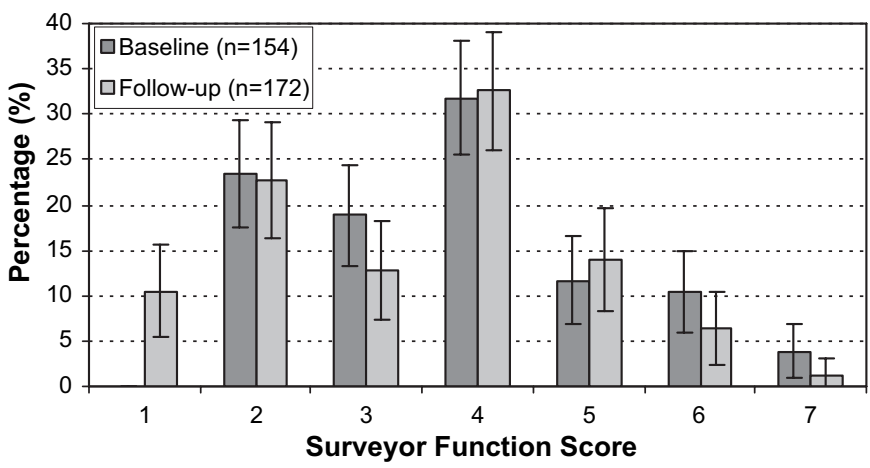

Figure 3 Distribution of Surveyor Function Score (with standard errors) for houses at baseline and follow-up.
Table 1 Percentage of houses failing to meet the infrastructure requirements for specific Healthy Living Practices (HLPs) included in the Failed Healthy Living Practice (FHLP) score for children at baseline and follow-up

\begin{tabular}{|c|c|c|c|}
\hline & Baseline & Follow-up & \multirow[b]{3}{*}{ p Value* } \\
\hline & ( $n=138$ houses) & $(n=153$ houses $)$ & \\
\hline & $\%$ & $\%$ & \\
\hline Wash clothes and bedding & 75.4 & 58.2 & 0.001 \\
\hline Prepare and store food & 81.9 & 66.0 & 0.002 \\
\hline Safe electrical & 44.2 & 20.9 & $<0.001$ \\
\hline Wash people & 87.7 & 65.4 & $<0.001$ \\
\hline $\begin{array}{l}\text { Remove human } \\
\text { waste/functioning toilet }\end{array}$ & 83.3 & 66.0 & 0.001 \\
\hline Remove waste water & 36.2 & 28.1 & 0.087 \\
\hline Remove rubbish & 82.6 & 77.8 & 0.189 \\
\hline Control dust (boundary fence) & 73.2 & 60.1 & 0.013 \\
\hline
\end{tabular}

*Fisher exact test $p$ value for one-tailed test (improvement in HLPs between baseline and follow-up).

improvements despite improvements in infrastructure. However, data from the study show only a weak association between crowding and hygiene (data not reported here), indicating that some households were able to achieve relatively good hygiene despite high levels of crowding; (3) the building programmes were based on reasonably rigorous standards ${ }^{30}$ and improvements were achieved across a wide range of components of housing infrastructure. Although these components of infrastructure are all recognised as important aspects of housing, their relative importance to health, and specifically to maintenance of domestic hygiene, is unclear.

Consideration of these issues raises two clear sets of implications for policy and further research. First, there is a need for better quality evidence on the relative health benefits of different components of housing infrastructure to support the design of houses that will result in the greatest potential health gain. The emphasis on achieving improvement across the full scope of building standards may limit the potential to achieve improvements in those components of infrastructure that may be most important to health. Housing programmes may need either to be massively scaled-up to provide housing to the current standard for new houses to a large proportion of the population in these communities or to take a more population-based approach, with a stronger emphasis on equity and achieving adequate standards for as many people as possible in aspects of infrastructure that are most essential to health improvement. High mobility of children between houses and/or households provides further justification for this approach, and is the rationale for the ecological analysis presented in this paper. The communities included in this study were the focus of the largest scale building programmes in 2004-5. Even with the large recent funding commitments to community housing programmes, ${ }^{31}$ significant increases in the proportion of the population of all communities beyond the scale of the building programmes in the study communities is unlikely. Policy makers may therefore need to compromise on standards of some aspects of infrastructure in order to build the large numbers of houses required to reduce crowding in communities and provide and maintain the essential infrastructure to enable achievement of domestic hygiene for all community residents.

Second, the lack of association between quality of infrastructure and hygiene, and the weak association between crowding and hygiene, highlight the need for hygiene promotion initiatives regardless of the quality of housing infrastructure or levels of crowding. Hygiene interventions such as hand washing 
Table 2 Mean score at baseline and follow-up for specific Healthy Living Practices (HLPs) included in the Surveyor Function Score (SFS) and Surveyor Condition Score (SCS)

\begin{tabular}{|c|c|c|c|c|c|c|}
\hline & \multicolumn{3}{|c|}{ Surveyor Function Score ( $1=$ best; $7=$ worst $)$} & \multicolumn{3}{|c|}{ Surveyor Condition Score ( $1=$ best; $7=$ worst $)$} \\
\hline & $\begin{array}{l}\text { Baseline } \\
(n=154)\end{array}$ & $\begin{array}{l}\text { Follow-up } \\
(n=172)\end{array}$ & Rank test p value* & $\begin{array}{l}\text { Baseline } \\
(n=154)\end{array}$ & $\begin{array}{l}\text { Follow-up } \\
(n=172)\end{array}$ & Rank test p value* \\
\hline Prepare and store food & $3.49(0.10)$ & $3.38(0.11)$ & 0.784 & $3.75(0.10)$ & $4.10(0.11)$ & 0.012 \\
\hline Wash clothes and bedding & $3.49(0.11)$ & $3.07(0.10)$ & 0.041 & $3.77(0.10)$ & $3.63(0.10)$ & 0.691 \\
\hline Wash people & $3.71(0.10)$ & $3.27(0.11)$ & 0.012 & $3.84(0.09)$ & $3.89(0.10)$ & 0.796 \\
\hline Remove human waste (toilet) & $3.56(0.10)$ & $3.13(0.10)$ & 0.020 & $3.93(0.11)$ & $3.92(0.11)$ & 0.761 \\
\hline Sleeping and bedding & $3.79(0.07)$ & $3.46(0.08)$ & 0.004 & $3.82(0.07)$ & $3.62(0.08)$ & 0.117 \\
\hline Control temperature & $3.88(0.08)$ & $3.52(0.08)$ & 0.001 & $3.97(0.07)$ & $3.81(0.07)$ & 0.087 \\
\hline Control dust & $3.95(0.09)$ & $3.78(0.07)$ & 0.321 & $3.92(0.08)$ & $3.78(0.07)$ & 0.101 \\
\hline Control mould & $3.77(0.09)$ & $2.98(0.10)$ & $<0.001$ & $3.88(0.10)$ & $3.44(0.09)$ & 0.001 \\
\hline House surrounds & $3.66(0.10)$ & $3.42(0.09)$ & 0.099 & $4.05(0.11)$ & $4.03(0.10)$ & 0.939 \\
\hline Separate animals and people & $3.95(0.05)$ & $3.66(0.08)$ & 0.003 & $3.97(0.09)$ & $3.91(0.09)$ & 0.515 \\
\hline Control pests and vermin & $4.01(0.10)$ & $3.41(0.10)$ & $<0.001$ & $4.20(0.11)$ & $3.90(0.10)$ & 0.028 \\
\hline Remove rubbish & $4.05(0.04)$ & $3.94(0.06)$ & 0.057 & $3.96(0.10)$ & $3.99(0.09)$ & 0.937 \\
\hline Reduce trauma & $3.62(0.11)$ & $3.39(0.12)$ & 0.130 & $3.94(0.10)$ & $3.84(0.11)$ & 0.403 \\
\hline Overall SFS/SCS & $3.75(0.11)$ & $3.38(0.12)$ & 0.057 & $4.19(0.12)$ & $4.20(0.12)$ & 0.870 \\
\hline
\end{tabular}

Data are shown as mean (SE).

*Kruskal-Wallis test of equality of proportions.

have been shown to be effective in reducing the incidence of a range of common infectious conditions among children. ${ }^{32} 33$ In resource-poor countries, an ecological approach to hygiene improvement that takes account of infrastructure, hygiene promotion and which provides an enabling environment has been recommended. ${ }^{34}$ However, evidence on the effectiveness of broader hygiene interventions in indigenous Australian communities $^{9}$ and internationally ${ }^{3536}$ is deficient, and there is a need for rigorous development and evaluation of such interventions.

Potential limitations of the study include: (1) reliability of infrastructure function and hygiene measures, although similar results from the different methods used and our repeatability analysis provides some reassurance on this point; (2) variation in the time between the surveys and the occupation of new houses. We aimed to conduct baseline surveys at least a few months before handover of completed houses to ensure that housing conditions were not allowed to deteriorate in anticipation of moving to a new house, and to conduct follow-up surveys at least several months after occupation of new houses to allow residents time to settle into routine living arrangements so housing conditions would reflect the impact of these arrangements. These time frames were usually achieved, although there were four communities where baseline surveys were conducted only 1-2 months before handover of new houses; (3) seasonal effects: Baseline and follow-up surveys were staggered over the

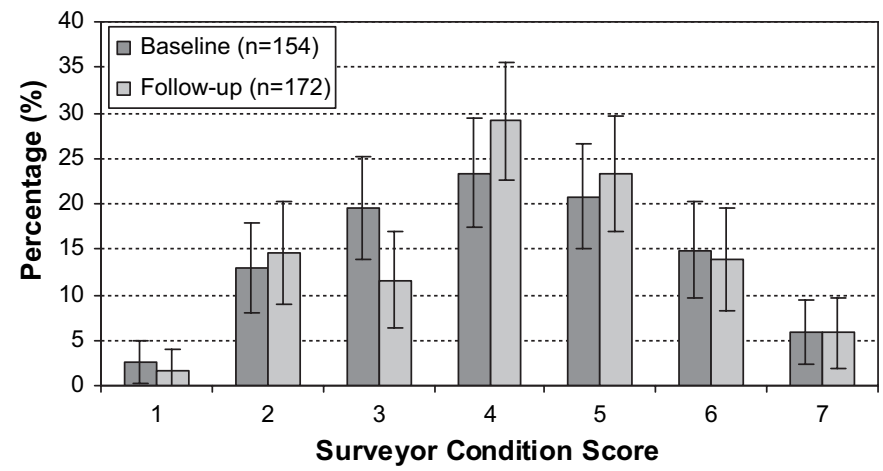

Figure 4 Distribution of Surveyor Condition Scores (with standard errors) for houses at baseline and follow-up. years and tended to be conducted around the same time each year, limiting the potential for any seasonal effects.

\section{CONCLUSION}

The scope and history of disadvantage of indigenous people in Australia means there are substantial political challenges to achieve agreement on the design and implementation of housing programmes. Regardless of house design, it is increasingly clear that the benefits of improved infrastructure are unlikely to be fully realised without concurrent hygiene promotion programmes as an integral part of a broader ecological approach to housing improvement. An ecological approach to housing-related interventions would use a multifaceted programme targeting priorities in key areas of influence: infrastructure, behaviour, access to cleaning equipment and providing a supportive policy environment. ${ }^{8} 37$

From a research/evaluation perspective, failure to demonstrate improvements in the health of individual children living in houses with improved infrastructure may be the result of failure to achieve improvements at the community level (as demonstrated here) rather than the lack of a causal association between quality of housing and child health. We will be reporting on this association at the individual child level in a separate paper, and the findings in relation to the community level impact will be important in interpretation of this analysis.

\section{What is already known on this subject}

- The general acceptance of the importance of housing to human health and well-being is reflected in the enshrinement of the right to adequate housing in international instruments. For decades, housing conditions have been identified as a major contributor to the poor health status of Australia's indigenous people. Despite health improvement being an explicit purpose of indigenous housing programmes, the housing conditions and health of many indigenous Australians continue to be very poor. 


\section{What this study adds}

- This study's findings show that housing programmes that are largely limited to improving the functional state of infrastructure have limited impact at the community level on major housing-related risks to health such as domestic hygiene. There was a marked improvement in the functional state of houses for some children but, at a population level, there appears to have been little change. The building programmes were based on reasonably rigorous standards and improvements were achieved across a wide range of components of housing infrastructure, all of which are believed to be important to health.

- This study highlights the need to gain a clearer understanding of the relative importance to health, and specifically to maintenance of domestic hygiene, of different components of housing infrastructure, of community level impacts of housing programmes and of the need for a stronger evidence base on interventions to create healthy household environments in disadvantaged settings.

Acknowledgements We wish to acknowledge the hard work and commitment of project and fieldwork officers Phil O'Donohoe (deceased), Kat Lonergan and Harold Ulamari. The participation of community residents and the assistance of the Indigenous Housing Association of the NT (IHANT), Community Housing and Community Council Officers is greatly appreciated.

Funding National Health and Medical Research Council, Canberra, Australia. Cooperative Research Centre for Aboriginal Health, Darwin, Australia.

Competing interests None

Ethics approval Ethics approval for the study was obtained from the Human Research Ethics Committees in the Top End and Central Australia of the Northern Territory. Informed written consent was obtained from everyone who participated in the study.

Provenance and peer review Not commissioned; externally peer reviewed.

\section{REFERENCES}

1. Department of Family and Community Services. Community Housing and Infrastructure Program (CHIP): program guidelines 2005-2006. Canberra: Australian Government, 2005.

2. McPeake T, Pholeros P. Fixing houses for better health in remote communities. In: Research and Analysis Branch of Department of Family CSalA, ed. Australian Social Policy 2006: improving the lives of Australians. Canberra: Australian Government, 2007:111-23.

3. National Aboriginal and Torres Strait Islander Health Council (NATSIHC). National strategic framework for aboriginal and Torres Strait Islander health: framework for action by Governments. Canberra: NATSIHC, 2003.

4. Australian Institute of Health and Welfare. Aboriginal and Torres Strait Islander Health Performance Framework 2008 report: detailed analyses. Canberra: AlHW, 2008. Report No.: Cat. no. IHW 22.

5. Australian Government/Northern Territory Government. Common Policy Framework: Northern Territory Indigenous Housing Program 2005-2008. Darwin: Northern Territory Government, 2005.

6. Pholeros P, Rainow S, Torzillo P. Housing for health: towards a healthy living environment for aboriginal Australia. Newport Beach, NSW: HealthHabitat, 1993.

7. Long J. The Commonwealth government and aboriginal housing, 1968-81. In: Read P, ed. Settlement: a history of Australian indigenous housing. Canberra: Aboriginal Studies Press, 2000:103-17.

8. Bailie RS, Wayte KJ. Housing and health in indigenous communities: key issues for housing and health improvement in remote Aboriginal and Torres Strait Islander communities. Aust J Rural Health 2006;14:178-83.
9. McDonald E, Bailie R, Brewster D, et al. Are hygiene and public health interventions likely to improve outcomes for Australian Aboriginal children living in remote communities? A systematic review of the literature. BMC Public Health 2008;8:153.

10. Cairnduff S, Guthridge S. Exploring indigenous home management programs in the Northern Territory. Darwin: Cooperative Research Centre for Aboriginal and Tropical Health, 2002.

11. Tatz C. Innovation without change. In: Hetzel B, ed. Health services for aborigines. St Lucia, Queensland: University of Queensland Press, 1974:107-20.

12. Wright J, Gundry S, Conroy R. Household drinking water in developing countries: a systematic review of microbiological contamination between source and point-of-use. Trop Med Int Health 2004;9:106-17.

13. Black M. Learning what works: a 20 year retrospective view on international wate and sanitation cooperation. Washington, DC: UNDP-World Bank Water and Sanitation Program, 1998.

14. de Bruijne G, Geurts M, Appleton B. Sanitation for all? Delft, The Netherlands: IRC International Water and Sanitation Centre, 2007.

15. Curtis VA. Dirt, disgust and disease: a natural history of hygiene. J Epidemiol Community Health 2007;61:660-4.

16. Shaw M. Housing and public health. Annu Rev Public Health 2004;25:397-418

17. Baker M, McNicholas A, Garrett N, et al. Household crowding a major risk factor for epidemic meningococcal disease in Auckland children. Pediatr Infect Dis $J$ 2000;19:983-90.

18. Fuller TD, Edwards JN, Sermsri S, et al. Housing, stress, and physical well-being: evidence from Thailand. Soc Sci Med 1993;36:1417-28.

19. Krieger J, Higgins DL. Housing and health: time again for public health action. $A m \mathrm{~J}$ Public Health 2002;92:758-68.

20. Sandel M, Wright RJ. When home is where the stress is: expanding the dimensions of housing that influence asthma morbidity. Arch Dis Child 2006:91:942-8.

21. Fien J, Charlesworth E, Lee G, et al. Towards a design framework for remote indigenous housing. Melbourne: AHURI, 2008, Report No. 114

22. Bailie RS, Stevens MR, McDonald E, et al. Skin infection, housing and social circumstances in children living in remote indigenous communities: testing conceptual and methodological approaches. BMC Public Health 2005:5:128.

23. Western Australian Aboriginal Child Health Survey. The social and emotional wellbeing of Aboriginal children and young people. Perth: Telethon Institute for Child Health Research, 2006.

24. Steering Committee for the Review of Government Service Provision. Overcoming indigenous disadvantage key indicators, 2007 report. Canberra: Commonwealth of Australia, 2008.

25. Australian Bureau of Statistics, Australian Institute for Health and Welfare The health and welfare of Australia's indigenous people, 2008. Canberra: ABS, 2008. Report No: Cat. no. 4704.0.

26. Larson EL, Aiello AE. Hygiene and health: an epidemiologic link? Am J Infect Control 2001;29:232-8.

27. Saegert SC, Klitzman S, Freudenberg N, et al. Healthy housing: a structured review of published evaluations of US interventions to improve health by modifying housing in the United States, 1990-2001. Am J Public Health 2003:93:1471-7.

28. Kowal E, Donohoe P, Lonergan K, et al. Dust, distance and discussion: fieldwork experiences from the Housing Improvement and Child Health Study. Journal of Australian Institute of Environmental Health 2005:5:59-72.

29. Aboriginal Health Strategy Unit. Environmental health standards for remote communities in the Northern Territory. Darwin: Northern Territory Government Environmental Health Task Group, Northern Territory Government, 2001.

30. Department of Family and Community Services. National indigenous housing guide. 2nd ed. Canberra: Commonwealth of Australia, 2003.

31. Council of Australian Governments. Fact sheet: National Partnership Agreement on Remote Indigenous Housing, Nov 2008. http://www.coag.gov.au/ coag meeting outcomes/2008-11-29/docs/

20081129 remote_indigenous_housing_factsheet.pdf (accessed Jun 2009)

32. Cairncross S, Curtis V. Hygiene and sanitation promotion. Geneva: Water Supply and Sanitation Collaborative Council, 2002.

33. Ejemot RI, Ehiri JE, Meremikwu MM, et al. Hand washing for preventing diarrhoea Cochrane Database Syst Rev 2008;(1):CD004265.

34. Storti C. The hygiene improvement framework: a comprehensive approach for preventing childhood diarrhea. Washington: Environmental Health Project, 2004. Joint Publication 8. http://www.ehproject.org/PDF/Joint Publications/JP008-HIF.pdf.

35. Fewtrell L, Kaufmann RB, Kay D, et al. Water, sanitation, and hygiene interventions to reduce diarrhoea in less developed countries: a systematic review and metaanalysis. Lancet Infect Dis 2005;5:42-52.

36. Aziz KM, Hoque BA, Hasan KZ, et al. Reduction in diarrhoeal diseases in children in rural Bangladesh by environmental and behavioural modifications. Trans $R$ Soc Trop Med Hyg 1990:84:433-8.

37. Bailie RS, Wayte KJ. A continuous quality improvement approach to Indigenous housing and health. Environ Health 2006:6:34-9. 